\title{
Comparison of Nonlinear Domain Decomposition Schemes for Coupled Electromechanical Problems
}

\author{
A. Halbach, P. Dular, C. Geuzaine \\ University of Liège, Department of Electrical Engineering and Computer Science, Liège, Belgium
}

\begin{abstract}
The aim of this paper is to compare several domain decomposition schemes for nonlinear, coupled electromechanical problems. Both staggered and monolithic electrostatic/elastic formulations are combined with an overlapping domain decomposition method applied either to the uncoupled, linear staggered resolutions or to the monolithic nonlinear system. The influence of the elastic waves frequency, of the electrostatic potential and of the mesh on the convergence rate is investigated on a simple $2 \mathrm{D}$ model of a vibrating micromembrane array.
\end{abstract}

Index Terms-Finite element analysis, Convergence of numerical methods, Nonlinear systems, Microactuators.

\section{INTRODUCTION}

Numerical simulation of many microelectromechanical systems (MEMS) involve a coupling between electrostatic fields and elastic deformations. Such systems might be way too large to solve with a direct solver at an affordable cost. Capacitive Micromachined Ultrasound Transducers (CMUT) for example consist in thousands of electrically actuated membranes forming a cell array, well suited for domain decomposition methods (DDM) [1]. Linear domain decomposition methods, such as letting a fixed-point, Gauss-Seidel or GMRES algorithm solve at every iteration the uncoupled, linear electrostatic and elasticity formulations, can be used to numerically simulate systems such as the CMUT. However, since the electroelastic coupling is nonlinear, an intrinsically non-linear approach to the domain decomposition method could also be explored.

This paper investigates the behavior of the convergence rate of these two families of DDM as the elastic wave frequency, the applied electrostatic potential and the mesh vary.

\section{ELECTROMECHANICAL FORMULATIONS}

Let $v$ be the electrostatic potential defined on region $\Omega=$ $\Omega_{e}, u$ the displacement field defined on region $\Omega_{m} \subset \Omega$ with components $u_{x}$ and $u_{y}$ and $M$ the mechanic 2D operator such that $M(u)=\left[\begin{array}{lll}\frac{\partial u_{x}}{\partial x} & \frac{\partial u_{y}}{\partial y} & \frac{\partial u_{x}}{\partial y}+\frac{\partial u_{y}}{\partial x}\end{array}\right]^{T}$. A region with a star superscript denotes a region deformed by the displacement field.

The electrostatic uncoupled weak formulation with no electric charges may be written as follows: Find $v$ such that

$$
\left\{\begin{array}{c}
-\int_{\Omega_{e}^{*}} \epsilon(\nabla v)^{T} \nabla v^{\prime} d \Omega=0, \\
v=\bar{v} \quad \text { on } \Gamma_{e}, \\
v=\bar{v}_{I} \quad \text { on } \Sigma_{e},
\end{array}\right.
$$

holds for appropriate test functions $v^{\prime}$, where $\epsilon$ is the electric permittivity. Defining $E_{x}=\frac{\partial v}{\partial x}$ and $E_{y}=\frac{\partial v}{\partial y}$ and using the Frobenius matrix product ' $:$ ' such that $A: B=\sum_{i, j} A_{i, j} B_{i, j}$ the 2D linear elasticity weak formulation is written as: Find $u$ such that

$$
\left\{\begin{array}{c}
\int_{\Omega_{m}} \frac{E}{1-\nu^{2}}\left[\begin{array}{ccc}
1 & \nu & 0 \\
\nu & 1 & 0 \\
0 & 0 & \frac{1-\nu}{2}
\end{array}\right] M(u)^{T} M\left(u^{\prime}\right) d \Omega \\
+\int_{\Omega^{*}} \frac{\epsilon}{2}\left[\begin{array}{cc}
E_{x}^{2}-E_{y}^{2} & 2 E_{x} E_{y} \\
2 E_{x} E_{y} & E_{y}^{2}-E_{x}^{2}
\end{array}\right]:\left[\begin{array}{ll}
\nabla u_{x}^{\prime} & \nabla u_{y}^{\prime}
\end{array}\right] d \Omega \\
-\int_{\Omega_{m}}^{\rho} \rho(2 \pi f)^{2} u^{T} u^{\prime} d \Omega=0, \\
u=\bar{u} \text { on } \Gamma_{m}, \\
u=\bar{u}_{I} \text { on } \Sigma_{m},
\end{array}\right.
$$

holds for appropriate test functions $u^{\prime}$, where $\nu$ is Poisson's modulus, $E$ Young's modulus and $\rho$ the volumic mass. The second term in 2 is the (nonlinear in $v$ ) electrostatic force computed using the virtual work principle, see e.g. [2].

These two formulations consider two sets of Dirichlet boundary conditions for the electric potential and the displacement field, respectively on $\Gamma_{e}, \Sigma_{e}$ and $\Gamma_{m}, \Sigma_{m}$. The boundaries $\Sigma_{e, m}$ are artificial interfaces between the overlapping electrostatic and mechanical regions used in the DDM to exchange information between the subdomains in the form of Dirichlet data $v_{I}, u_{I}$. (More sophisticated coupling schemes will be considered in the full paper.)

The staggered coupling consists in solving the electrostatic and elasticity formulations (1)-(2) in alternance. The monolithic coupling consists in solving (1)-(2) at once, using either a fixed-point or a Newton-Raphson scheme. For the latter, the linearized coupling terms have to be computed to obtain the Jacobian matrix [3]. In all cases a classical first-order nodal finite element discretisation is used [4].

\section{DDM FOR ELECTROMECHANICAL COUPLING}

Combining domain decomposition with the above coupled electromechanical problem can be achieved in multiple ways. The DDM iteration can be performed using a fixed point (FP) or a Gauss-Seidel (GS) iteration when applied to a nonlinear 


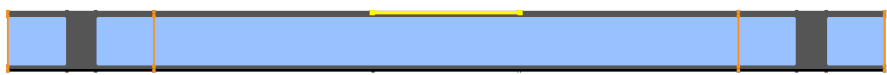

Figure 1. One of the two subdomains in the micromembrane geometry.

problem as well as any iterative linear solver (e.g. GMRES [5][6]) when applied to a linear problem. The multiphysics coupling can be handled using a Newton-Raphson (NR) iteration for the monolithic formulation as well as the staggered (STAG) iteration for the uncoupled one.

We focus here on the following OuterLoop(InnerLoop) combinations:

1) $\{$ FP,GS $\}(\mathrm{NR}) \rightarrow$ Fixed Point/Jacobi or Gauss-Seidel outer DDM iteration, each nonlinear subproblem being solved in a monolithic way using Newton-Raphson;

2) $\{$ FP,GS $\}($ STAG) $\rightarrow$ Fixed Point/Jacobi or Gauss-Seidel outer DDM iteration, each nonlinear subproblem being solved in a staggered way;

3) STAG(GMRES) $\rightarrow$ GMRES inner DDM iteration on the linear electrostatic and elastic formulations; Outer staggered iteration for the multiphysics coupling;

4) NR(GMRES) $\rightarrow$ GMRES inner DDM iteration on the linear electrostatic and elastic formulations; Outer NR iteration for the multiphysics monolithic coupling;

\section{NumericAl Tests}

We consider a simple 2D model of a vibrating micromembrane array. Two subdomains are modeled in order to evaluate the cross coupling between two adjacent membranes in the array (one subdomain is depicted on Figure 1). A membrane has the following characteristics: length $60 \mu \mathrm{m}$, support pillars thickness $2 \mu \mathrm{m}$, membrane thickness $0,3 \mu \mathrm{m}$, bulk thickness $0,3 \mu \mathrm{m}$, electrode (yellow) length $10 \mu \mathrm{m}$, solid domain (grey) with $\epsilon=3,9 \cdot 8,854 \cdot 10^{-12} \mathrm{~F} / \mathrm{m}, E=150 \cdot 10^{9} \mathrm{~N} / \mathrm{m}^{2}, \nu=$ 0,3 and air domain (blue) with $\epsilon=8,854 \cdot 10^{-12} \mathrm{~F} / \mathrm{m}$. The orange lines represent the artificial interfaces $\Sigma_{e, m}$ between the overlapping subdomains (overlap length is $5 \mu \mathrm{m} \approx 8 \%$ ). Homogeneous Dirichlet boundary conditions are applied on the whole bottom (black) line for both electrostatic and elastic subproblems. Tests were performed for several electrode-toground voltages below pull-in voltage. The test frequencies were selected so as to avoid resonances.

Figure 2 displays the number of outer and inner iterations for both FP(NR) and STAG(GMRES), the same electrodeto-ground over pull-in voltage ratio being used for every frequency. $\mathrm{FP}(\mathrm{NR})$ converges slowlier for frequencies higher than about $48 \mathrm{MHz}$ (corresponding roughly to 4 elastic wavelengths per subdomain). At $61 \mathrm{MHz}$ and higher it diverges, which might be linked to the (simple) Dirichlet interface conditions for DDM. The linear approach to DDM on the other hand does not show any significant slowdown in the given frequency range. In addition to the iteration count, the cost of one FP(NR) inner multiphysics iteration is significantly higher than for the outer iterations of STAG(GMRES), which makes it uncompetitive in this particular test case. One can also notice how the number of multiphysics iterations is almost independent of the frequency in the considered range.
Figure 3 displays the number of iterations of the multiphysics loop for both FP(NR) and STAG(GMRES) for a given frequency $(48 \mathrm{MHz})$ when the applied electrode voltage is increased. The iteration count for $\mathrm{FP}(\mathrm{NR})$ is fairly stable with increasing voltage, whereas STAG(GMRES) shows a significant increase.

Further details about the coupling schemes will be provided in the full paper.

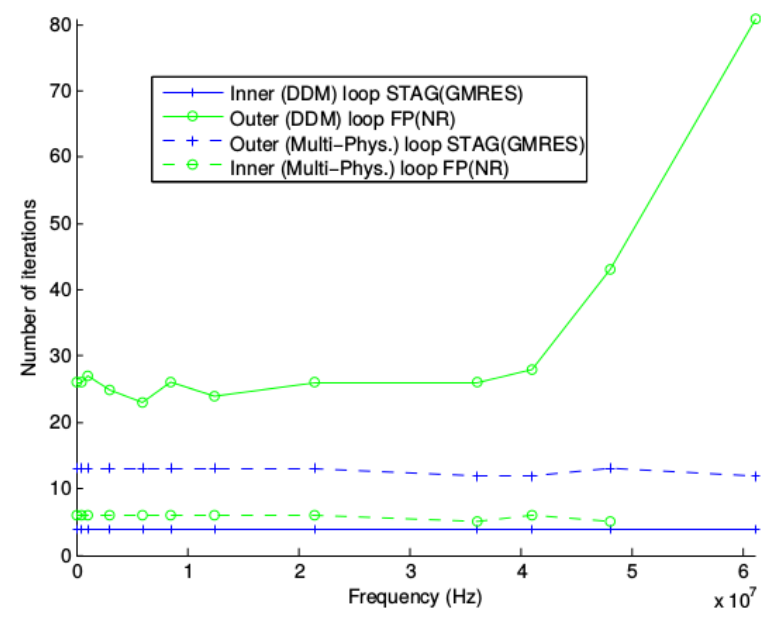

Figure 2. Max number over all test voltages of the inner and outer loops for methods $S T A G(G M R E S)$ and $F P(N R)$ versus frequency $(H z)$.

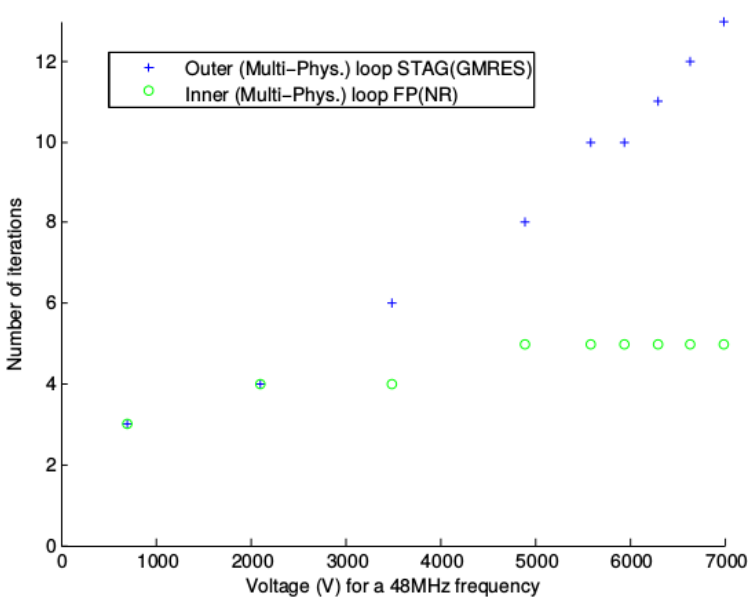

Figure 3. Number of multiphysics iterations for $48 \mathrm{MHz}$.

\section{REFERENCES}

[1] A. Toselli and O. Widlund. Domain decomposition methods - algorithms and theory. Ser. Comput. Math. Springer, 2005.

[2] J. L. Coulomb. A methodology for the determination of global electromechanical quantities from a finite element analysis and its application to the evaluation of magnetic forces, torques and stiffness. IEEE Transactions on Magnetics, 19(6):2514-2519, 1983.

[3] V. Rochus, D. J. Rixen, and J.-C. Golinval. Monolithical modeling of electro-mechanical coupling in micro-structures. International Journal for Numerical Methods in Engineering, 65(4):474-482, 2006.

[4] O. C. Zienkiewicz, R. L. Taylor, and J. Z. Zhu. The Finite Element Method: Its Basis and Fundamentals. Butterworth-Heinemann, 2013.

[5] Y. Saad. Iterative Methods for Sparse Linear Systems. Society for Industrial and Applied Mathematics, second edition, 2003.

[6] A. Vion and C. Geuzaine. Double sweep preconditioner for optimized Schwarz methods applied to the Helmholtz problem. J. Comput. Phys. 266:172-174, June 2014. 